\title{
Desenvolvimento de software educacional livre e inclusão de alunos com deficiência visual
}

\author{
Mírian Bruckschen ${ }^{1}$, Sandro José Rigo ${ }^{1,2}$, Édina Fagundes ${ }^{1}$ \\ ${ }^{1}$ Universidade do Vale do Rio dos Sinos - UNISINOS \\ ${ }^{2}$ Universidade Federal do Rio Grande do Sul - UFRGS \\ cleo.sl@gmail.com, rigo@unisinos.br, evfagundes@unisinos.br
}

\begin{abstract}
Resumo: O desenvolvimento de software educacional que proporcione acessibilidade aos alunos com necessidades educacionais especiais é uma necessidade facilmente identificável no contexto atual. Entretanto, a grande maioria das aplicações conhecidas não atende aos requisitos de acessibilidade, e algumas das soluções disponíveis com este objetivo possuem um custo proibitivo e muitas vezes não são adequadas às situações vivenciadas pelos alunos que as utilizam. Neste artigo, apresentamos um relato de desenvolvimento de software educacional acessível e livre, voltado para inclusão de pessoas com deficiências visuais. Para tal desenvolvimento, houve a interação de uma equipe multidisciplinar envolvendo inclusive os alunos e seus professores, além de profissionais da Pedagogia e Informática.
\end{abstract}

Palavras-chave: deficiência visual, educação, tecnologia, software livre

\section{Development of free educational software for inclusion of students with visual impairments}

\begin{abstract}
Development of educational software that provides accessibility to the students with special educational needs is an easily noticeable issue nowadays. However, most of known applications does not support accessibility requirements, and some of the available solutions are too expensive and often aren't suitable to the situations lived by these students. In this paper, we present a development report of a free (open source) accessible educational software, targeted at children with visual impairments. This process counted with the participation of a multidisciplinary team, including the children and their teachers, professionals of Pedagogy and Informatics.
\end{abstract}

Keywords: visual impairment, education, technology, free software

\section{Introdução}

O perfil dos usuários que se beneficiam dos recursos tecnológicos é cada vez mais heterogêneo, dado a crescente popularização e disponibilização de alternativas, variando desde computadores e celulares, caixas eletrônicos e sofisticados sistemas de som e vídeo. Exemplos desta diversificação dos usuários de computadores, por exemplo, incluem crianças ainda não alfabetizadas, pessoas idosas e pessoas com diferentes tipos de deficiências ou necessidades especiais. Esta ampliação no uso de recursos tecnológicos estende-se às escolas, inclusive naquelas voltadas para alunos com necessidades educacionais especiais. Entretanto, a grande maioria dos dispositivos tecnológicos envolvem interação e uso de mais de um sentido (visão, audição, cognição), fato que determina a dificuldade ou impossibilidade para que parte deste público potencial possa fazer uso adequado destes dispositivos (Preece et al., 1994; Booth, 1989).

Atualmente, já existem recursos disponíveis para proporcionar com qualidade adequada a síntese de voz, a partir de conjuntos de textos que podem ser gerados 
dinamicamente. Esta disponibilidade tecnológica proporciona o suporte necessário para o desenvolvimento de aplicações educacionais que atendam aos requisitos de acessibilidade para o um público com deficiência visual. Entretanto, muitas das aplicações conhecidas são caras e desenvolvidas de modo um tanto alheio às reais necessidades dos seus usuários.

Desta forma, objetivando o encaminhamento de uma solução que possibilite o desenvolvimento de aplicações educacionais com uso de síntese de voz em software livre e cujo projeto e avaliação incorpore a participação efetiva de seus futuros usuários, apresentamos o relato do projeto, implementação e teste de uma aplicação educacional acessível, realizada de forma participativa, em um contexto de uma equipe multidisciplinar de desenvolvimento, voltada para alunos do ensino fundamental com deficiências visuais.

Os principais diferenciais deste trabalho consistem em seu caráter multidisciplinar e na interação com os usuários finais desde a sua concepção. Isso possibilitou, acreditamos, melhores resultados e uma contribuição mais completa e rica ao público a que se destina e à sociedade como um todo.

\section{Deficiência, acessibilidade e inclusão}

Importante fator motivador para o crescente interesse na área de acessibilidade é a chamada inclusão social, cuja história é bastante recente. No início da década de 80 , começou a surgir um movimento pela inclusão de pessoas com deficiências na sociedade, que logo foi denominado desta forma (Werneck, 1999; Sassaki, 1997; Sidarta, 2003). (Sidarta, 2003) define inclusão como a "inserção total e incondicional" do indivíduo com deficiência na sociedade.

O Brasil apresenta um quadro preocupante frente à várias demandas sociais. Destacamos como problemática as diversas contingências que fragilizam à acessibilidade à educação para todos. Nosso país possui $\mathbf{3 3}$ milhões de analfabetos funcionais (cerca de $18 \%$ da população), ou seja, pessoas com menos de quatro anos de estudo, e 16 milhões de pessoas com mais de 15 anos que ainda não foram alfabetizadas (IBGE, 1999).

Considerando este contingente de adolescentes e adultos analfabetos funcionais, matriculados ou não, há ainda a problemática de que, entre eles, há ainda aqueles demandatários de educação inclusiva. Estes, por certo, apresentam peculiaridades acentuadas em sua forma e tempo de aprender, possibilidades visuais, auditivas, motoras, entre outras.

(LOPES, 2006, p.8) afirma que:

"Tratar a questão da inclusão pela diferença não significa anular a diferença ou não; pelo contrário, pode significar, entre outras coisas, redimensioná-la. Significa pensar a diferença dentro de um campo político, onde experiências culturais e comunitárias e práticas sociais são colocadas como integrantes da produção dessas diferenças."

Neste universo, há um evidente contingente não alfabetizado, mesmo tendo freqüientado a educação especial, buscado a rede regular ou a suplência, ainda carregam a sequiela do analfabetismo. Entre os analfabetos há aqueles que nunca se aproximaram da escola, mesmo porque ainda é vivo o pré-conceito de que "deficientes" ficam em casa e não precisam de estudo.

Para aqueles(as) estudantes com deficiência, há a previsão legal de incluí-los(as) nas redes regulares de ensino. O que defendemos é que tal política educacional, constituída majoritariamente à revelia do ethos da alteridade deficiente, formatada em prescrições legais, pouco pode resultar naquilo que diz pretender. A acessibilidade 
pedagógica e arquitetônica precisa acontecer com o desenvolvimento tecnológico e com a participação efetiva dos sujeitos a quem se destina, pois não nos parece tão natural construirmos nossos fazeres profissionais, hegemonicamente, da lógica da normalidade para a deficiência.

Cumpre indagar ainda: como aprenderão se não forem compreendidos em sua identidade e cultura? Aqueles que nunca ou pouco freqüientaram a escola e têm sobre si a prescrição da normalidade, apresentam ainda assim sua própria e inerente demanda, indicando que há urgências a serem investigadas e respondidas, junto com estes sujeitos.

Nesse complexo relacional os alunos com deficiência, neste caso aqueles com deficiência visual, compõem aquele grupo que demanda voz para que sejam possíveis reflexões, posturas, metodologias, habilidades, para experiência de seus direitos educacionais, para além da acessibilidade aparente. Acessibilidade com construções participativas, que considerem suas peculiaridades, desejos e potencialidades.

Pensar a acessibilidade, neste caso, acessibilidade educacional, implica em buscarmos coletivamente alternativas de permanência e sucesso escolar, tanto mais para os cidadãos em condições que incitam os conceitos de fracassados sociais por não terem acesso à alfabetização efetiva, suporte para infinitas aprendizagens e exercício de cidadania.

O que não podemos subjugar, por isso reiteramos, é que nada disto resultará em possibilidade de acessibilidade e inclusão se não nos dermos conta que qualquer tentativa de promoção de acessibilidade, será menos acessível, se construída desde o lugar da normalidade, que quer algo para este diferente, silenciando-o na gênese daquilo que oferece o eixo da construção a ser materializada.

Eis aí o sentido inegociável da construção de UMA SOLUÇÃO PARA POSSIBILITAR O ACESSO A UM AMBIENTE DE JANELAS EM GNU/LINUX POR PESSOAS COM NECESSIDADES ESPECIAIS: tecnologias a serviço da aprendizagem inclusiva, ter-se dado COM os sujeitos da pretendida acessibilidade à própria ferramenta, como potência a outras tantas que lhe são de direito.

\section{Visão geral da solução}

A solução baseou-se no sistema operacional GNU/Linux e na distribuição Ubuntu ${ }^{1}$, que é uma distribuição simples e intuitiva, voltada para as necessidades do usuário final. Esta escolha facilita a possível adoção da solução em contextos onde a aquisição de licenças de software representaria um custo proibitivo. Além disso a utilização de uma plataforma livre permite que a solução seja expandida, no caso de uma efetivação de equipes de colaboradores. Para o desenvolvimento da aplicação utilizam-se recursos como o ambiente de janelas do sistema operacional e suporte para tecnologias assistivas, a AT-SPI ${ }^{2}$ (Assistive Technologies Service Provider Interface). O componente conhecido como sintetizador de voz transforma um texto gerado dinamicamente em componentes de áudio, usando regras de fonética ou combinando arquivos de som pré-gravados.

Uma das etapas mais importantes deste projeto foi a definição deste ferramental a ser utilizado pela solução de acessibilidade proposta. Antes da preparação do ambiente e implementação da solução e do protótipo, um tempo considerável foi investido no teste de leitores de tela e sintetizadores de voz para a definição de quais destas ferramentas seriam utilizadas no projeto.

No que se refere a leitores de tela, foram estudadas três alternativas gratuitas. São elas: o MBROLA ${ }^{3}$, o Gnopernicus ${ }^{4}$ com o banco de dados desenvolvido pela equipe da UFRGS ${ }^{5}$ e baseado nas bibliotecas OGI $^{6}$, e o GNOME Orca ${ }^{7}$.

A utilização do MBROLA foi descartada devido a sua licença bastante restritiva, 
mesmo para usuários domésticos e organizações sem fins lucrativos. Isto impede que seja distribuído livremente por "empacotadores" de distribuições de sistemas livres como o GNU/Linux ${ }^{1,8}$, e inviabiliza seu uso neste projeto. Estas restrições eram conhecidas desde antes dos testes, mas achamos interessante a execução deste teste para fins de comparação com as demais aplicações leitoras de tela.

Entre a seleção do Gnopernicus ${ }^{4}$ e $\mathrm{Orca}^{7}$, foi realizada uma pesquisa sobre a facilidade de instalação e continuidade de manutenção destas aplicações. Dessa forma, foi percebido que o Gnopernicus tem caído em desuso e sido substituído pelo Orca na maioria das distribuições GNU/Linux. Além disso, se mostrou de difícil instalação. Apesar de a configuração não ser complicada, ele oferece menos recursos que o seu substituto Orca. Já o Orca se mostrou bastante fácil de instalar e configurar, e possui uma comunidade de desenvolvedores e usuários ativa e comprometida com o projeto. Por ser um projeto recente (possui versões para usuários finais faz cerca de um ano), não existe muita documentação disponível, o que é um ponto contra a sua utilização. Entretanto, isso tem mudado rapidamente nos últimos meses, o que tende a melhorar com a sua utilização como padrão em distribuições GNU/Linux populares como o Ubuntu (a partir da versão 6.10, Edgy Eft ${ }^{9}$ ).

Também foram realizados testes com diversos sintetizadores de voz para o idioma português, tais como Festival ${ }^{10}$, Mbrola $^{3}$ e eSpeak ${ }^{11}$. Os resultados mais interessantes foram obtidos com os sintetizadores Festival e eSpeak, sendo que estes resultados estão disponíveis para download ${ }^{12}$. Devido aos resultados apresentados, à simplicidade de configuração e uso do eSpeak, foi decidido por sua utilização neste projeto.

\subsection{Projeto da interface e análise de usuários}

A interface de comunicação da solução com a participação do usuário final pode ser descrita pelas seguintes etapas, que ocorrem de forma cíclica:

1) o usuário interage com a aplicação através de dispositivos comuns de entrada, como o mouse e o teclado;

2) a solução de acessibilidade baseada em voz sintetiza o comando ou opção selecionada pelo usuário e o reproduz através do sistema de som do computador.

Os usuários da solução podem ser divididos em dois grandes grupos bemdefinidos: alunos em fase de alfabetização com deficiências e professores destes alunos. Todos os alunos têm algum tipo de dificuldade, em maior ou menor grau, no desempenhar de alguma atividade. Em se tratando de alunos com necessidades especiais, como deficiência visual, cognitiva e motora, essas dificuldades são mais flagrantes, e freqüentemente mais difíceis de contemplar. Além disso, existem dificuldades conhecidas quanto a tempo e recursos para a formação dos professores.

Aprofundamos esta análise após visitas à unidade sede dos testes deste projeto, a Escola Estadual de Ensino Fundamental Marechal Ilha Moreira, localizada em São Leopoldo/RS, que também trabalha com a formação inclusiva de crianças e adolescentes com deficiências visuais. Com esta aproximação, objetivamos a participação destes alunos e professores no processo de construção dessa solução, envolvendo os usuários em todas as etapas, desde a sua concepção.

Com base na avaliação destes grupos, foram definidos alguns dos requisitos da interface: simples e intuitiva, porém interessante, colorida (para que os alunos pequenos que não possuam qualquer deficiência visual tenham motivação para explorar a aplicação) e com os resultados dos comandos e seleções sendo disponibilizados através de som e não somente através de imagens, para que os alunos com deficiência visual possam utilizá-la. 
Foram feitas observações das aulas, do uso que os referidos alunos fazem do computador e das dificuldades apresentadas. Com base nestas observações foi desenvolvida uma atividade para estimular os princípios de contagem e aritmética básica, utilizando a infra-estrutura já mencionada. A principal motivação está ligada ao fato de que as atividades que são apresentadas pelo DOSVOX, aplicação utilizada atualmente na escola, se baseiam na "tabuada" (DOSVOX 2001a, DOSVOX 2001b), privilegiando a memorização em detrimento do raciocínio lógico-matemático.

\section{Prototipação e testes}

Com base nos requisitos relacionados e na necessidade de um desenvolvimento rápido e que possibilitasse a fácil extensão posterior, optou-se pela linguagem de scripts Python $^{13}$, que suporta Orientação a Objetos e oferece suporte à interface GTK+, através do módulo PyGTK ${ }^{14}$. Além disso, é uma linguagem de aprendizado rápido e fácil, e fornece módulos prontos e embutidos na linguagem para diversas aplicações.

O protótipo tem uma classe bastante importante, que é a classe que instancia a aplicação: MathStoryGame, no arquivo prototipo.py. Os demais arquivos-fonte em Python (extensão .py) disponibilizam funções auxiliares e constantes utilizadas nas funções principais. $\mathrm{O}$ arquivo XML prototipo.glade representa as interfaces utilizadas na aplicação, e foi criado a partir da ferramenta de construção de GUIs em GTK+ Glade $^{15}$.

A Figura 1 apresenta uma visão geral do protótipo na linguagem de modelagem UML, com a classe principal e demais arquivos fonte com funções auxiliares. Nota-se nesta visão apresentada a descrição previamente detalhada: uma classe principal (MathStoryGame), que interage e se baseia nas demais classes auxiliares de constantes, definição de histórias na forma de dicionários do Python a serem utilizados na classe principal da aplicação, métodos de interface e outras funções utilitárias. Também estão destacados no diagrama os principais métodos que descrevem a interação da aplicação: startGame, loadStoryWindow, nextScene, playStoryGame, e métodos de interação com o usuário através do teclado. 


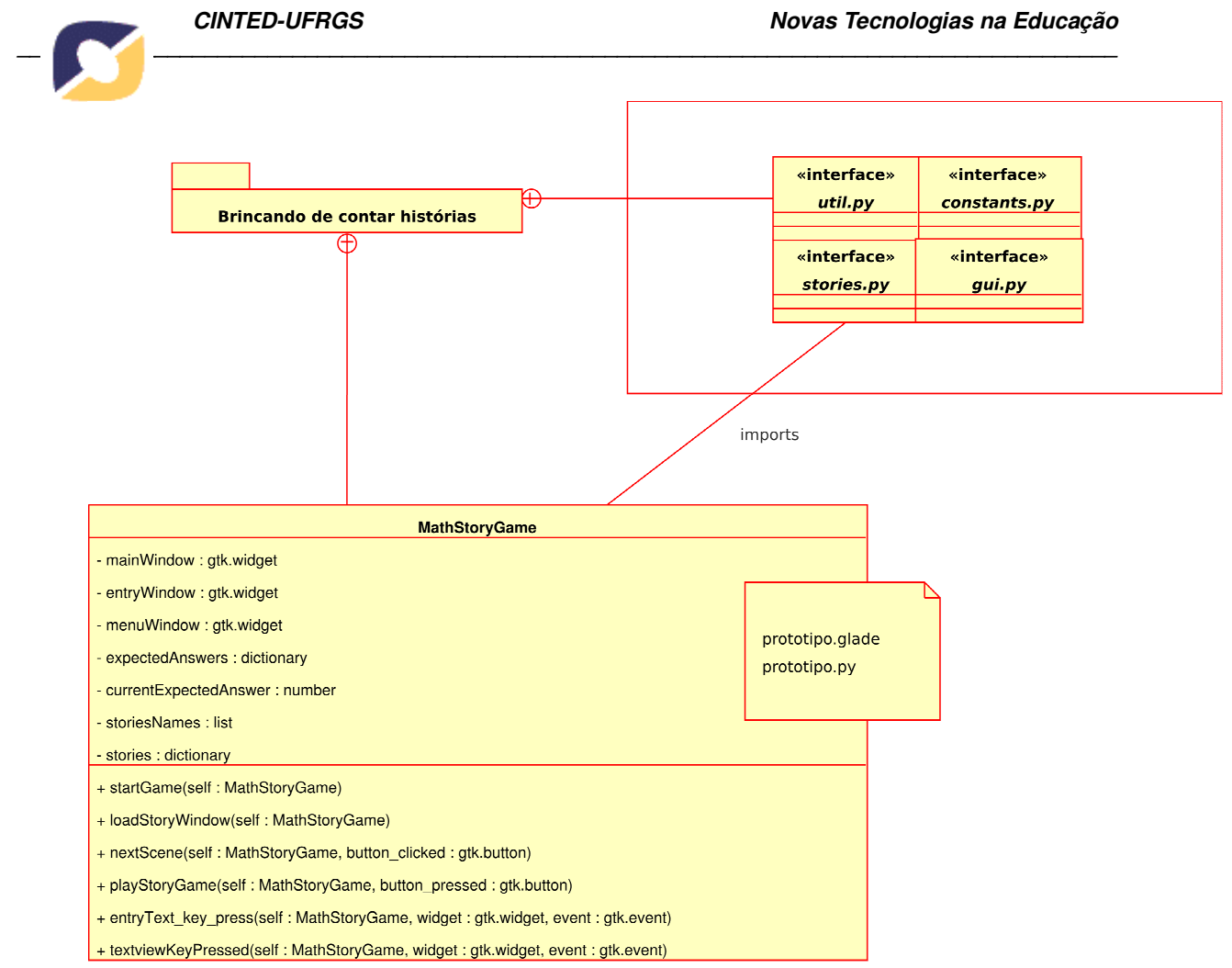

Figura 1: Diagrama UML apresentando a visão geral do protótipo

A interação do usuário com a aplicação ocorre fundamentalmente pelo teclado, dadas as limitações do principal público-alvo no tocante à manipulação do cursor na tela, operação orientada basicamente pela visão.

São apresentadas cenas de histórias matemáticas (que podem ser estendidas e configuradas facilmente), com texto, imagem ilustrativa e, opcionalmente, questões sobre as histórias. Na configuração das histórias são suportadas funcionalidades como a escolha aleatória de elementos de narrativa, para que não sejam contadas as histórias sempre da mesma forma (um exemplo seria a contagem de "animais convidados para a festa" na história prototipada "A festa dos bichos", que pode variar de acordo com a vez que a aplicação é executada), escolha de respostas (como na questão "Quantos jogadores existem num time de futebol?" na história "Um passeio ao estádio") e escolha das demais operações matemáticas básicas (soma, subtração, divisão e multiplicação).

Sobre a utilização de imagens nas histórias do protótipo, o principal objetivo do é incluir como público-alvo também crianças sem deficiências visuais ou com deficiências leves ou moderadas. Dessa forma, a aplicação não exclui grupo algum de alunos com a deficiência como fator limitador, objetivando ser realmente inclusiva.

A Figura 2 mostra a tela de abertura do protótipo em sua versão atual, com a listagem das duas histórias disponibilizadas. Os elementos da figura 2 mostram uma interação (percorrendo os elementos da esquerda para a direita, de cima para baixo) em uma cena da história matemática "A festa dos bichos".

As histórias disponibilizadas inicialmente tiveram como inspiração a interação inicial com a escola onde a aplicação foi testada e em entrevistas com os alunos e suas professoras. As questões destas histórias foram formuladas para alunos dos primeiros anos do ensino fundamental. 


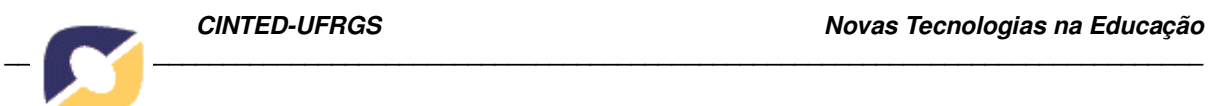

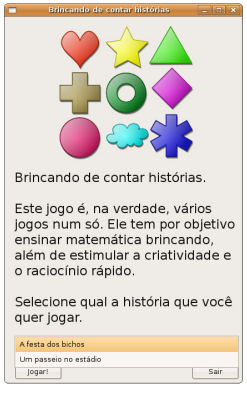
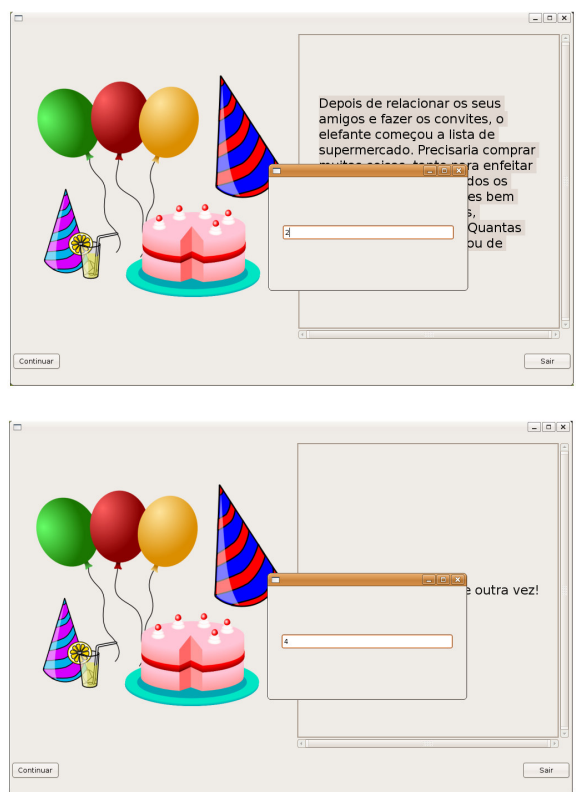
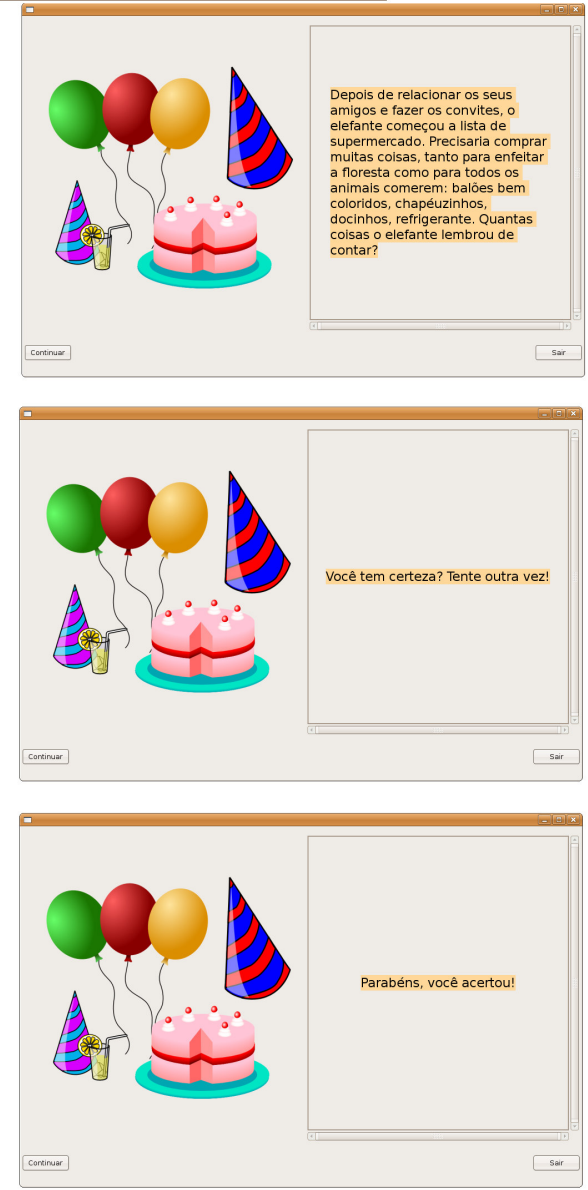

Figura 2: Interação com a aplicação

Durante os testes, foram consideradas principalmente os seguintes itens: i) entendimento das sentenças sintetizadas pelo eSpeak através do Orca; ii) usabilidade do protótipo; c) se as funcionalidades propostas originalmente foram atendidas.

Os principais requisitos se mostraram atendidos amplamente: o entendimento e o fluxo da aplicação se mantiveram contínuos, com interferências apenas nas primeiras execuções (para apresentação do funcionamento da aplicação, principalmente); e os usuários se mostraram extremamente interessados, em especial devido ao cuidado tomado com a temática das histórias utilizadas na aplicação.

\section{Considerações finais}

O atual desenvolvimento tecnológico deve ser colocado a serviço ético da qualidade de vida de todas as pessoas, sendo que a acessibilidade pode auxiliar a viabilizar a inclusão social e educacional, entre outras. A necessidade de trabalho de projeto e desenvolvimento envolvendo ações profissionais interdisciplinadas e a participação efetiva e intransferível das pessoas com deficiência foi o objetivo do trabalho apresentado neste artigo.

Objetivos como propostos por (Delors et al., 1996) e (Morin, 2000) para uma educação que contribua com a implementação de uma sociedade efetivamente inclusiva para serem alcançados, demandam a utilização de recursos tecnológicos de forma integrada com propostas pedagógicas inclusivas, a partir do desenvolvimento de soluções envolvendo a participação daqueles demandatários parcialmente atendidos, 
silenciados historicamente, sejam pessoas com deficiências ou com dificuldades de aprendizagem. Pensamos que aplicações educacionais têm por obrigação serem disponibilizadas a todos os públicos, não sendo a deficiência o limitador para o uso e aprendizado dos alunos com a utilização de tecnologia.

Durante os testes preliminares executados na escola junto aos alunos e professoras, nossas opiniões iniciais de que o caminho certo para o desenvolvimento de ferramentas (quaisquer, mas educacionais em particular) deve ter seus requisitos elaborados a partir da interação com o público-alvo de destino se confirmaram, dada a importância desta interação para a definição e ajustes do trabalho desenvolvido.

Os testes têm mostrado resultados bastante interessantes e entendemos que poucos ajustes separam a aplicação de ser uma ferramenta bastante poderosa nas mãos de educadores do ensino fundamental - e mesmo médio, com as devidas adaptações no protótipo - e ela tem diferenciais importantes, especialmente nos dias de hoje: sua construção e, hoje, utilização em conjunto com os sujeitos educativos com deficiências mostra a sua aplicabilidade na prática para este público-alvo.

A acessibilidade é um objetivo que todos os desenvolvedores e pesquisadores da atualidade devem buscar. Soluções acessíveis, inclusivas a todos os públicos, só trazem benefícios à educação e, conseqüentemente, a toda sociedade.

\section{Notas de texto}

1. http://www.ubuntu.com/

2. http://developer.gnome.org/projects/gap/

3. http://tcts.fpms.ac.be/synthesis/mbrola.html

4. http://www.baum.ro/gnopernicus.html

5. http://www.cslu.ogi.edu/tts/

6. http://www.cslu.ogi.edu/corpora/spoltech/

7. http://live.gnome.org/Orca

8. http://www.debian.org/social_contract\#guidelines

9. https://wiki.ubuntu.com/EdgyReleaseNotes

10. http://www.cstr.ed.ac.uk/projects/festival/

11. http://espeak.sourceforge.net/

12. http://mirian.is.dreaming.org/tecsoc2007/teste1.wav e http://mirian.is.dreaming.org/tecsoc2007/teste1b.wav; http://mirian.is.dreaming.org/tecsoc2007/teste2.wav e http://mirian.is.dreaming.org/tecsoc2007/teste2b.wav

13. http://www.python.org/

14. http://www.pygtk.org/

15. http://glade.gnome.org/

\section{Referências}

BOOTH, Paul. An Introduction to Human-Computer Interaction. Hove, UK: Lawrence Erlbaum Associates, 1989.

DELORS, Jacques; AL MUFTI, In'am; ISAO, Amagi; CARNEIRO, Roberto; CHUNG, Fay; GEREMEK, Bronislaw; GORHAM, William; KORNHAUSER, Aleksandra; MANLEY, Michael; PADRÓN QUERO, Marisela; SAVANÉ, Marie Angélique; SINGH, Karan; STAVENHAGEN, Roberto; MYONG WON SUHR; ZOU NANZHAO. Educação: um tesouro a descobrir - Relatório para a UNESCO da Comissão Internacional sobre Educação para o século XXI. UNESCO no Brasil, 1996.

DOSVOX. Ações concretas através do sistema DOSVOX. 2001a. Disponível em: 
http://intervox.nce.ufrj.br/dosvox/acoes.html. Acesso em: julho de 2007.

DOSVOX. CONTAVOX: O Jogo das continhas. 2001b. Disponível em: http://intervox.nce.ufrj.br/dosvox/manuais/Contavox.txt. Acesso em: julho de 2007.

IBGE - INSTITUTO BRASILEIRO DE GEOGRAFIA E ESTATÍSTICA. 1999. Educação e condições de vida - Educação 1999. Disponível em: http://www.ibge.gov.br/home/estatistica/populacao/condicaodevida/indicadoresminimos tabela3.shtm. Acesso em: outubro de 2007.

LOPES, Maura Corcini. Problematizando os discursos que constituem a metanarrativa da inclusão escolar. Disponível em: http://www.humanas.unisinos.br/siapea/textos.htm. Acesso em: outubro de 2007.

MORIN, E. Os sete saberes necessários à educação do futuro. UNESCO no Brasil, 2000.

PREECE, J.; ROGERS, Y.; SHARP, H.; BENYON, D.; HOLLAND, S.; e CAREY, T. HumanComputer Interaction. Wokingham, England: Addison-Wesley, 1994.

SASSAKI, R. K. Inclusão: Construindo uma sociedade para todos. Rio de Janeiro, RJ, Brasil: WVA, 1997.

SIDARTA, Veev. Mídia e Deficiência. Brasília, DF, Brasil: Fundação Banco do Brasil, 2003.

WERNECK, Cláudia. Sociedade Inclusiva. Quem cabe no seu TODOS? Rio de Janeiro, RJ, Brasil: WVA, 1999. 